\title{
Enhanced-DSR: A New Approach to Improve Performance of DSR Algorithm
}

\author{
${ }^{1}$ Sharmin Sultana, ${ }^{2}$ Salma Begum, ${ }^{3}$ Nazma Tara, ${ }^{4}$ Ahsan Raja Chowdhury \\ Department of Computer Science \& Engineering, University of Dhaka, Dhaka, \\ Bangladesh \\ jui_sharmin@yahoo.com, ${ }^{2}$ jennycsdu@yahoo.com, ${ }^{3}$ taranaz2003@yahoo.com, \\ ${ }^{4}$ farhan717@cse.univdhaka.edu
}

\begin{abstract}
High overhead involved in flooding while route creation is a limiting factor of Dynamic Source Routing (DSR) protocol applied for mobile ad hoc networks (MANETs). During data delivery, it seems that we fully benefit from DSR if the route is not long enough. In this paper, we propose two modifications of DSR algorithm to enhance its performance. In the modified approach of DSR, to reduce broadcasting overhead, multicasting approach is used. Again, for shortening packet length, in case of longer route, the route is truncated after a predetermined number of hops. Some simulations show that the new modified algorithm (Enhanced DSR) performs better than the DSR algorithm.
\end{abstract}

\section{KEYWORDS}

DSR, E-DSR, AODV, RREP and RREQ.

\section{INTRODUCTION}

A mobile ad-hoc network (MANet) [1] is a kind of wireless ad-hoc network, and is a selfconfiguring network of mobile routers (and associated hosts) connected by wireless links - the union of which form an arbitrary topology[1].

Along with recent development of the ad hoc networks, their expected size will become large. It is thus required to assure the management of the large ad hoc networks efficiently. Many routing protocols have been proposed for the ad hoc networks. They are classified into plane routing protocols and hierarchical routing protocols [2]. As shown in Fig. 1, the plane routing protocols are composed of table driven (Proactive) and source-initiated on-demand driven (Reactive) protocols [3].

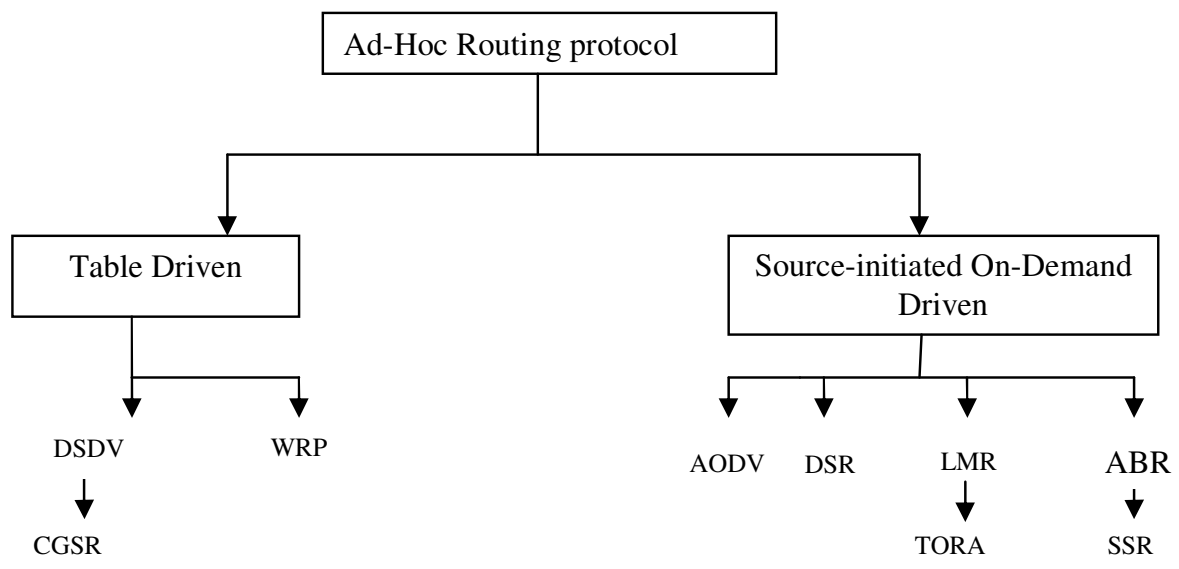

Figure 1. Categorization of Ad-Hoc Routing Protocol 
In the on-demand routing protocols, a route to a destination is required only when there is a data packet to deliver to that destination. In principle, each source broadcasts route request packets in the network to find the route. In DSR [4], when the destination receives the request packet, it sends back the route reply packet containing the complete route from the source to the destination along the reverse route to the source.

High overhead, involved in flooding during route creation, is a limiting factor of Dynamic Source Routing (DSR) protocol when applied for mobile ad hoc networks (MANETs). This paper analyzes through simulating an improvement of basic DSR to enhance its performance. In the Enhanced-DSR (E-DSR), to reduce Route Request Packet overhead, multicasting approach is used using Route Record field of Route Request option. Again, in case of longer route in the packet header, the route is truncated after a predetermined number of hops to shorten the packet length.

This paper consists of five sections. Section 2 describes definitions and literature review. Section 3 focuses on proposed idea. Section 4 presents experimental results and discussions and Section 5 concludes the paper.

\section{DEFINITIONS AND LITERATURE REVIEW}

The Dynamic Source Routing protocol allows mobile sources to discover paths towards any desired destination dynamically $[4,5]$. Every data packet includes complete list of nodes, which the packet must pass before it reaches the destination. Hence, all nodes that forward or overhear these packets may store routing information for future use. DSR can support fast network topology changes and service even asymmetric links; it can successfully find paths and forward packets in unidirectional link environments. Moreover, like AODV [6], it has a mechanism for on-demand route maintenance, so there are no periodic topology update packets. When link failures occur, only those nodes which forward packets through those links must receive proper routing advertisements. In addition, DSR allows source nodes to receive and store more than one path towards a specific destination. Intermediate nodes have the opportunity to select another cached route as soon as they are informed about a link failure.

When a source that desires to send data to a particular destination, first checks to whether it has a route in its cache for that destination. If it does, it will use that route by placing (in the data packet header) the sequence of hops that the packet must follow to reach the destination. If there is no such route stored in the local cache, then the source will initiate a new path discovery process, by broadcasting a Route Request to its neighborhood. This message contains the source and destination addresses, a request ID and an ordered intermediate node's address list, through which this message has passed. This node list is initially blank when the message leaves the source node (it has not yet visited any other node). Thereafter, every other node that receives this request message parses it to see if it is the intended destination. Every node processes the request according to the following steps:

1. If the initiator's address for this route request is found in this host's list of recently seen requests, then discard the route request packet and do not process it further.

2. If this host's address is already listed in the route record in the request, then discard the route request packet and do not process it further.

3. If the target of the request matches this host's own address, then the route record in the packet contains the route by which the request reached this host from the initiator of the route request. Return a copy of this route in a route reply packet to the initiator.

4. Otherwise, append this host's own address to the route record in the route request packet, and re-broadcast the request. 


\section{AN ENHANCED - DSR}

In dynamic source routing, when a host needs a route to another host, it dynamically determines one based on cached information and on the results of a route discovery protocol. Due to the source routing characteristic [5] of DSR, overhead is increased. Moreover, during the route discovery process, each node takes part in forwarding Route Request (RREQ) packet [7, 8]. Each node except the intended destination forwards the Route Reply (RREP) packet [7,8] to create the route. Though, these RREPs increase the number of multiple paths to reach destination, they increase the control packet load of the network. Our proposal is to modify the basic DSR to reduce the redundant RREPs and the control packet overhead.

\subsection{Reduction of Control Packet Overhead}

In Basic DSR, any host discovers a route to any other host through route discovery method. A host initiating a route discovery process, multicast a route request packet RREQ. When the host for which the route is requested receives the route RREQ packets, sends a route reply packet to the sender.

In DSR, to discover a route from source to destination, source broadcasts the RREP packets to its neighbors (hosts within its wireless transmission range). DSR protocol maintains a neighbor table to keep track of neighbors. Now if in an average each node has $\mathrm{x}$ neighbors and there are $\mathrm{y}$ hops from source to destination and $\mathrm{R}$ is the total no. of RREQ packets then basic DSR requires,

$$
\mathrm{R}=\mathrm{xy} \text { RREQ packets to be broadcasted. }
$$

This broadcast of RREQ packets imposes a higher degree of overhead in DSR.

To overcome the problem of flooding in DSR, multicast of RREQ packet is preferable to broadcast which has been proposed in E-DSR. When a node X receives a RREQ packet, it will perform following steps:

1. X finds out all of its neighbors from its neighbor table.

2. X selects addresses of those nodes from neighbor table which are not present in the Route Request option [4].

3. X forwards RREQ packets to the nodes found in step 2.

For example, a wireless network consists of 11 mobile nodes. Source $\mathrm{S}$ broadcasts to two nodes as like DSR. In DSR when RREQ packet reaches node $A$ of degree 5 in, it performs broadcasting to its neighbor node, but in E-DSR it sends RREQ packets to 4 nodes because when A receives RREQ from $S$ it appends its own address and the source route becomes [S, A]. Now A has 5 neighbors in its neighbor table S, C, B, E, and F. But since S is in source route, A won't send RREQ to $S$ which reduces one packet overhead. Similarly, B reduces one more packet overhead, E reduces one. In a total, DSR sends 31 RREQ packets and in the same case E-DSR sends 20 RREQ packets. As a result, E-DSR reduces control packet overhead to a great extent. This overall procedure of E-DSR is shown step by step in Figure 2. 


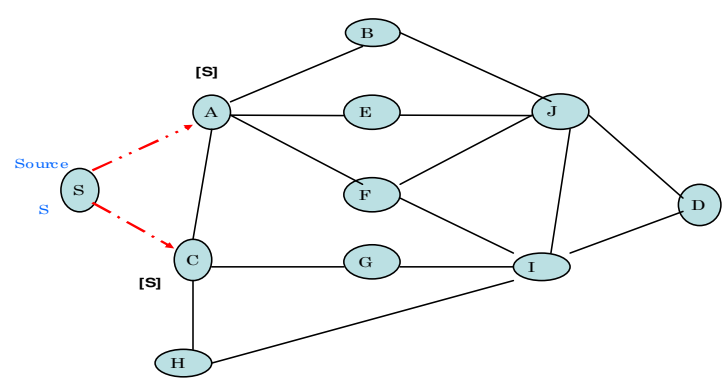

(a)Source node sending RREQ packets to its neighbors

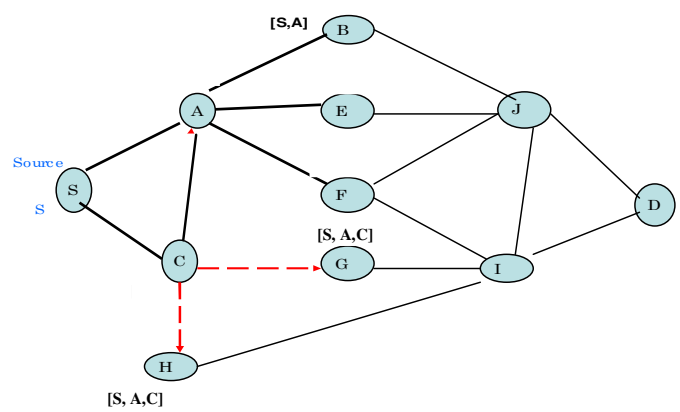

(c)Node c is transmitting RREQ packets. As node A and S are in it's node list, it doesn't send the packet to those node and so reduces 2 transmission.

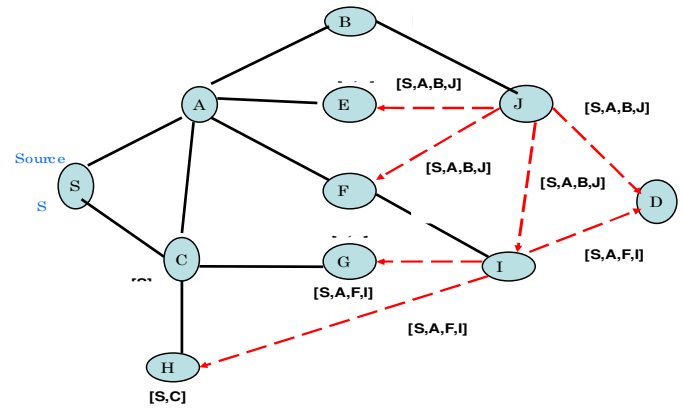

(e)Node $\mathrm{J}$ and I are sending the RREQ packets and intended destination has been found.

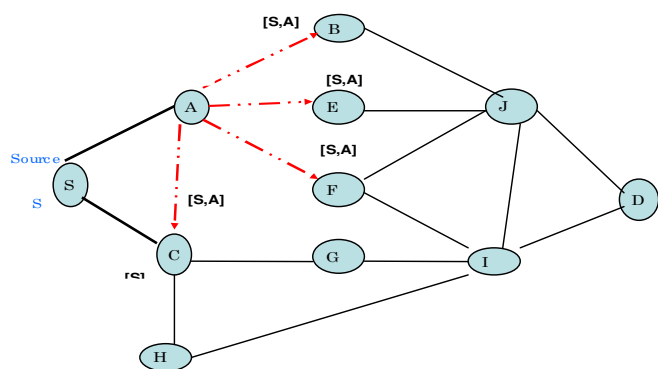

(b)Node A is sending RREQ packets to its neighbor except to $\mathrm{S}$. Thus reduces One RREQ packet transmission

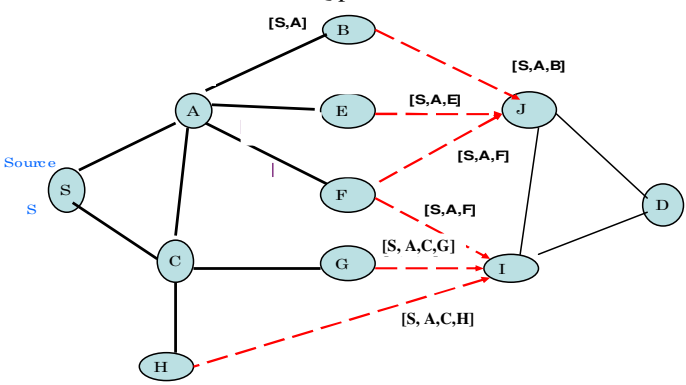

(d) Node B,E,F,G and $\mathrm{H}$ who have already got the RREQ packets now forwarding the RREQ packets in the same fashion

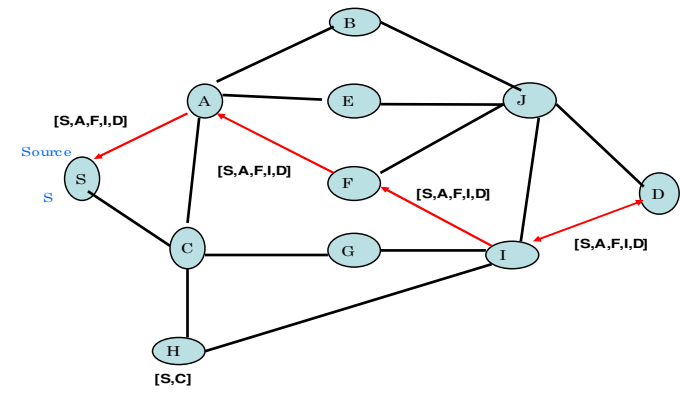

(f) Destination node is sending RREP packet to the source with the discovered route.

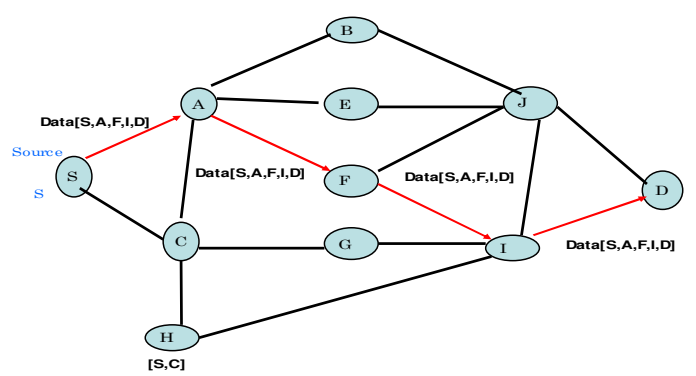

(g)Finally source node is transferring data packet to the destination node

Figure 2 (a-g). Steps in Reduction of Route Request Packet Overhead for a network having 11 nodes. 


\subsection{Reduction of Packet Header Length}

To send a packet to another host, the sender sends the source route in the packet's header, giving the address of each host in the network through which the packet should be forwarded in order to reach the destination host [4]. The sender then transmits the packet over its wireless network interface to the first hop identified in the source route. When a host receives a packet, if this host is not the intended destination of the packet, it simply transmits the packet to the next hop identified in the source route in the packet's header. Once the packet reaches its final destination, the packet is delivered to the network layer software on that host.

Each data packet sent from source to destination carries in its header the complete, ordered list of nodes through which the packet must pass. As a result, when the route length grows the packet header size also grows. DSR is suited for small to medium sized networks as its overhead can scale all the way down to zero $[4,5,8]$. The overhead will increase significantly for networks with larger hop diameters as more routing information will be contained in the packet headers.

After the route discovery when sender founds a complete route to the destination in its cache it can count the total number of hop in the route [9-11]. Based on the hop count source will select a value for TTL field.

According to the proposal of reducing packet header length if number of hops is less than 5, then it will work like traditional DSR. Otherwise the optimization technique will be applicable. Value of the TTL field depends on the total number of nodes in the route. The TTL field usually controls the maximum number of hop visited by the data packet. This TTL value is decremented by 1 as the data packet visits each hop.

Let source $\mathrm{S}$ finds the value for TTL is $\mathrm{x}$. Then if $\mathrm{x}$ is grater than 5, then sets the TTL value to 4 . Source will send the data packet with the entire source route in the data packet header and with the TTL value 4 . The source simply transmits the packet to the next hop identified in the source route in the packet's header. In the third hop which has TTL value 1, modify the data packet and then forwards the packet. Modifications of the data packet are as follows:

1. Truncate the route from the first node of route record to third node (Let Q).

2. Store the truncated route in Q's cache /or in some memory other than cache for a longer time period.

3. Send the data packet with route record from $\mathrm{Q}$ to the following nodes after reinitializing TTL to 4.

4. Repeat step 1 to 3 until it reaches the desired destination.

For example, Figure 3 illustrates an example of the procedure, in which a node $\mathbf{S}$ is attempting to send data packet to node $\mathrm{D}$. S sends data packet to $\mathrm{E}$ with route record $[\mathrm{S}, \mathrm{A}, \mathrm{E}, \mathrm{H}, \mathrm{I}, \mathrm{K}, \mathrm{M}$, $\mathrm{N}, \mathrm{D}]$. Node $\mathrm{A}$ and node $\mathrm{E}$ just forward the packet but node $\mathrm{H}$ which has TTL value 1 shrinks the route from [S, A, E, H, I, K, M, N, D] to [H, I, K, M, N, D] and forwards the packet after reinitializing TTL to 4 . The node $\mathrm{H}$ caches route $[\mathrm{S}, \mathrm{A}, \mathrm{E}, \mathrm{H}]$. Now from $\mathrm{H}$ when the packet reaches $M$ it again re-initialize $T T L=4$ and forwards the packet. When the packet reaches $D$ it keeps the packet because it is the intended destination and passes the packet to the upper layer. 


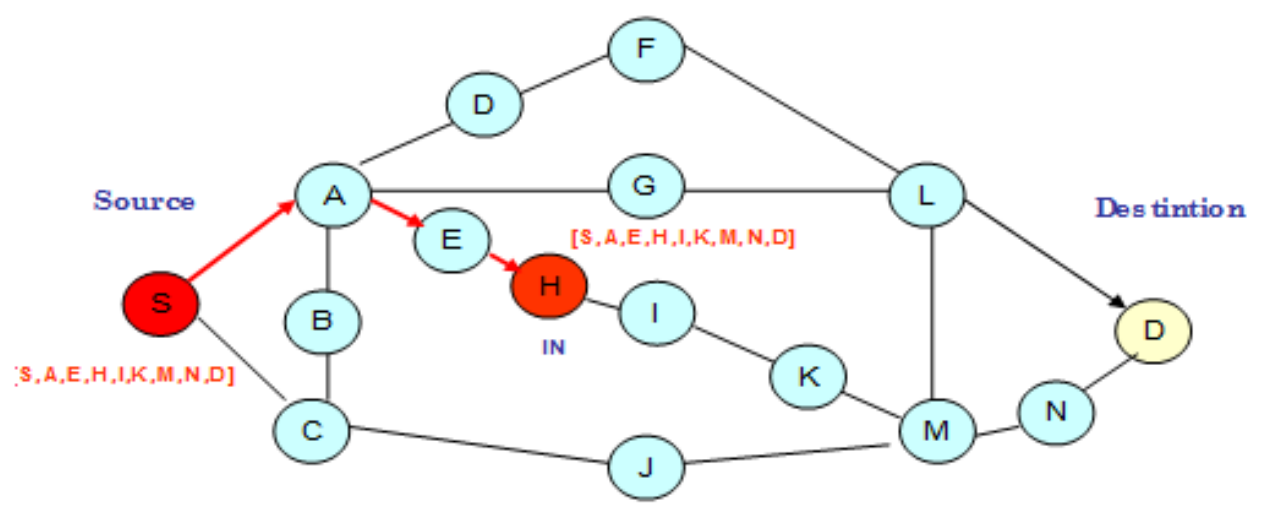

(a) Source node $\mathrm{S}$ has a predetermined route to which it is going to send data and the node has TTL value greater than 5 . So it will set TTL value to 4

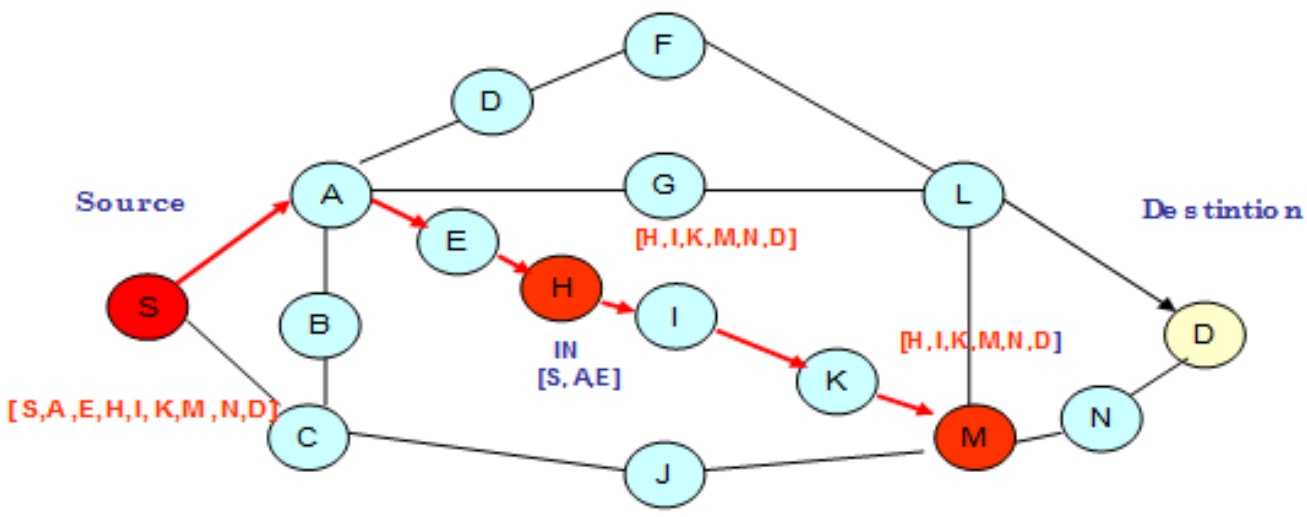

(b) Node H got TTL value 1 so it will truncate the route from S to E and forward the remaining list rather the entire list and at the same time it will also set TTL value to 4.

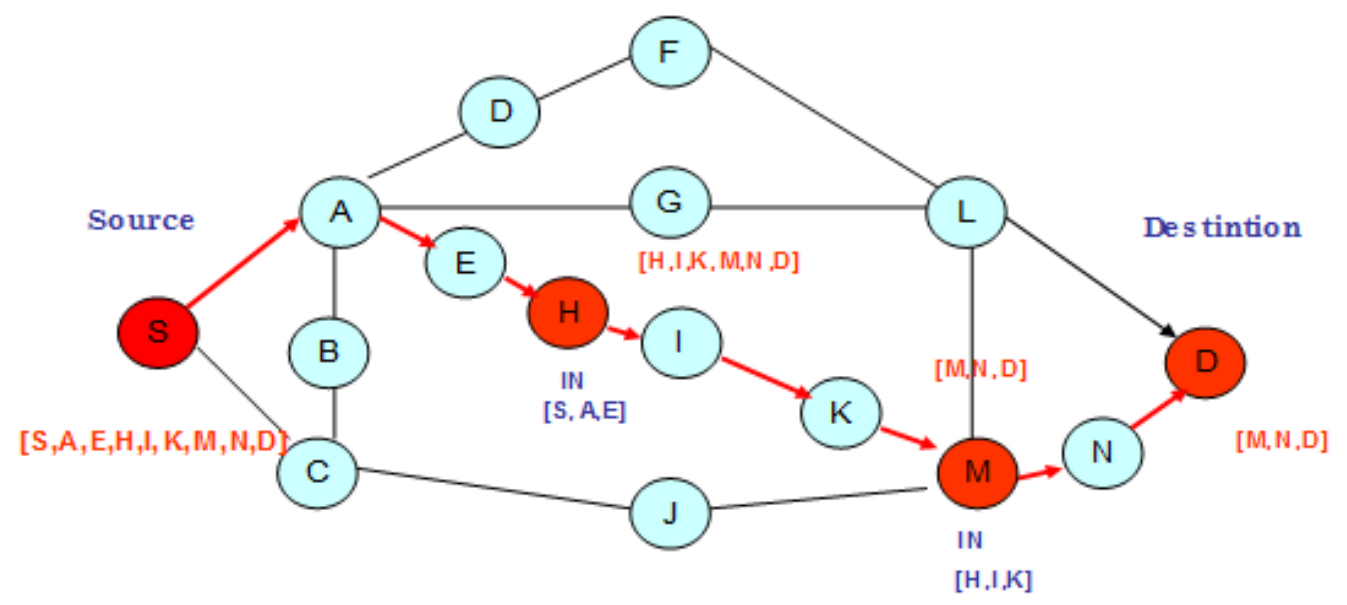

(c) Node M again found TTL value 1 and it will repeat the procedure.

Figure 3 (a-c). Steps in Reducing Packet header Length

The flow chart of E-DSR is shown in Figure 4 and 5. 


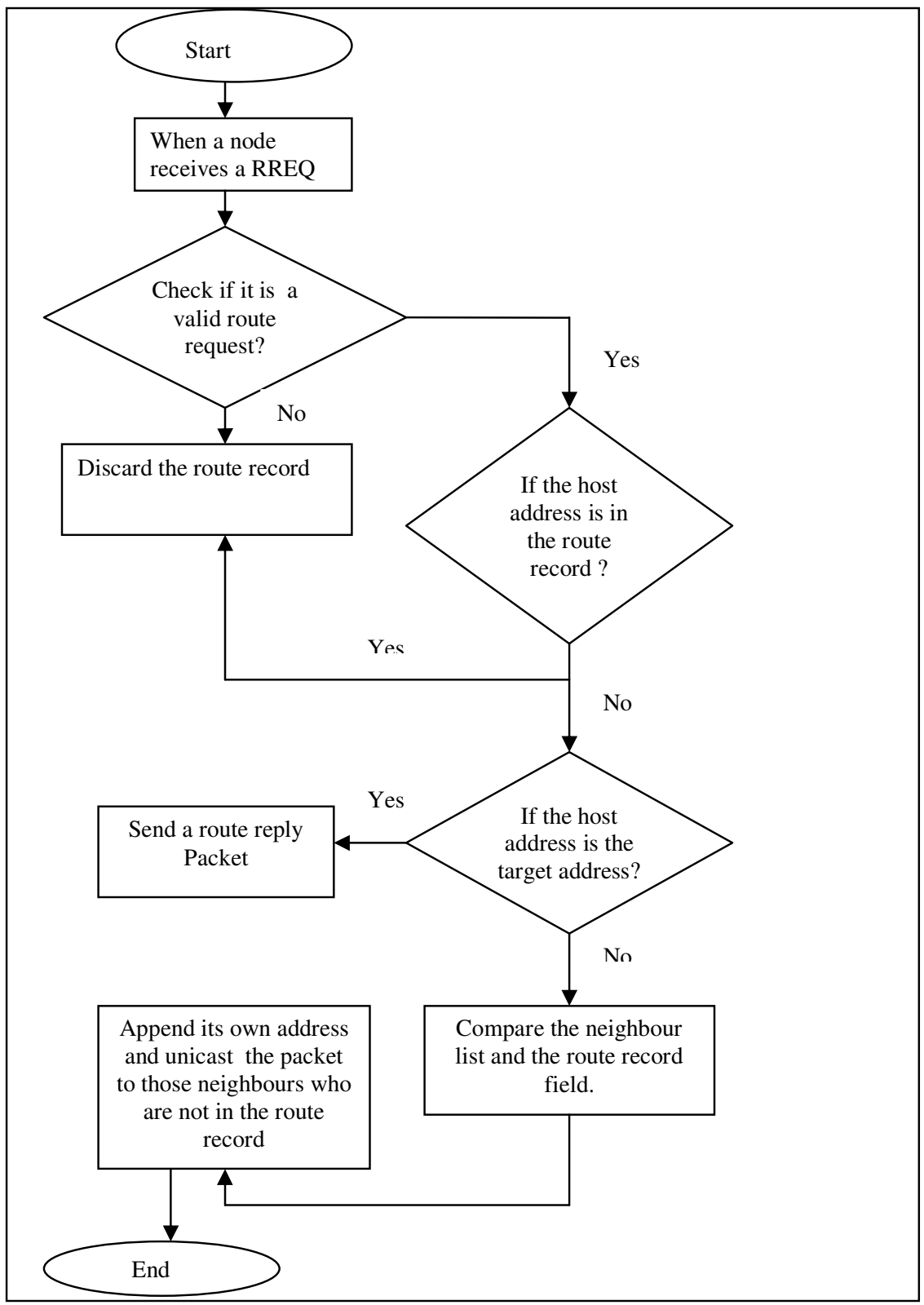

Figure 4. Flowchart of E-DSR reducing Route Request Packet

\section{SimUlation AND RESULT}

In E-DSR, the basic problem of DSR has been improved by reducing Route Request overhead and shortening packet length which has been shown by using the extended version of ns2 (network simulator version 2) [12]. In the simulation, it has been shown that the performance of E-DSR is much better than DSR.

Here it is assumed that, the ad-hoc network consists of 100 wireless nodes, moving about over a $1500 \times 1000$ flat space for 200 seconds of simulated time. In order to enable direct, fair comparisons between the DSR and the E-DSR, it was critical to challenge the protocols with identical loads and environmental conditions. Each run of the simulator accepts a scenario file as input that describes the exact motion of each node and the exact sequence of packets 


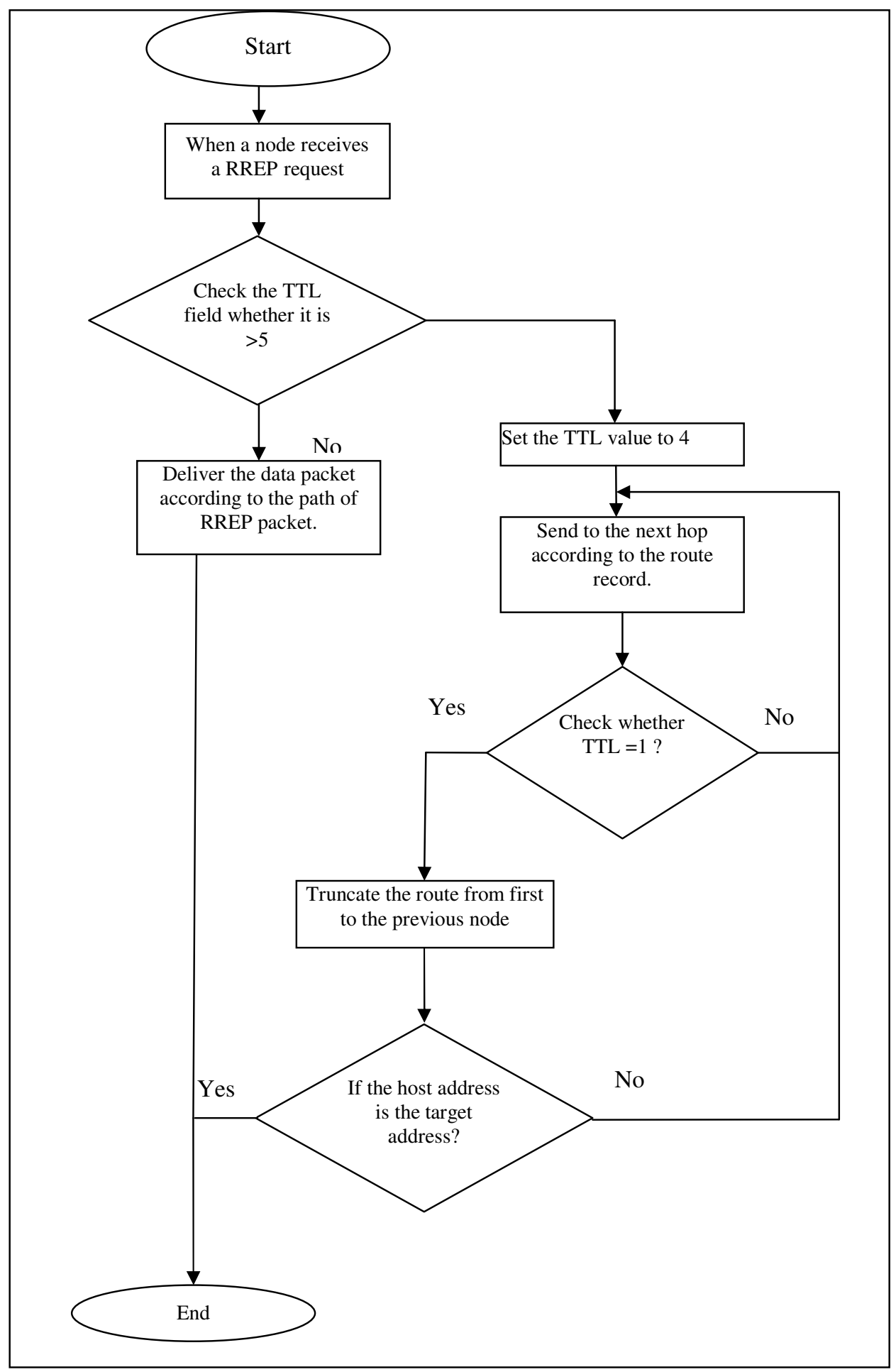

Figure 5. Flowchart of E-DSR shortening packet header length

originated by each node, together with the exact time at which each change occurs in motion or packet origination. Since each protocol was changed in an identical fashion, the performance of these protocols can directly be compared. 


\subsection{Route Request Packet Overhead}

For the same network E-DSR reduces broadcasting of packets. Figure 6. reveals this concept. From the graph, it can be concluded that, as the number of nodes increases in a network, number of broadcast packet is reduced in ESDR than that of DSR.

\subsection{Control Packet Overhead}

In DSR, broadcasting of each Route request packet involves several consecutive control packets like RTR, MAC [4, 7]. So, reduction of Route Request packets in E-DSR also reduces control packet overhead (shown in Figure 7)

\subsection{Data Delivery Ratio}

Delivery ratio is a measure of efficiency. In E-DSR, since the number of control packet is reduced, number of sent packet is also reduced. So there is less traffic in the network and delivery ratio is high. So it can be said that, E-DSR is more efficient than DSR. From Figure 8. , this improvement can be observed.

Figure 6 and 7 are the comparison results in Route Request packet overhead and Control packet overhead between DSR and E-DSR for networks with 10, 25, 50, 75 and 100 nodes. Figure 8 is the performance comparison of DSR and E-DSR in Data delivery ratio.

\section{CONCLUSION}

This paper has proposed modified DSR algorithm E-DSR with two new concepts: Reducing Route Request packet and Truncating the packet header length in DSR. Performance of E-DSR is elevated in respect of some simulation metrics such as Route Request and control packet overhead, and packet delivery ratio. Unlike other source routing protocols, the E-DSR adapts quickly to routing changes by reduction of sending route request packet as well as shortening the packet length when the size of the wireless network is large enough.

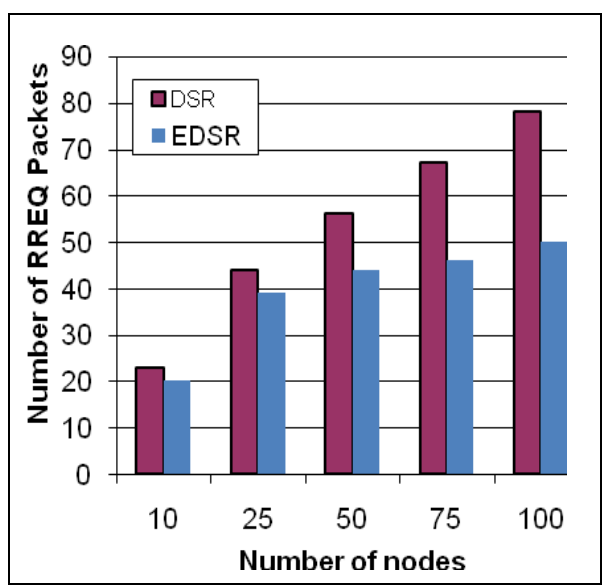

Figure 6. Comparison of DSR and E-DSR in case of reducing RREQ packets for a network of 10 , 25, 50, 75 and 100 nodes.

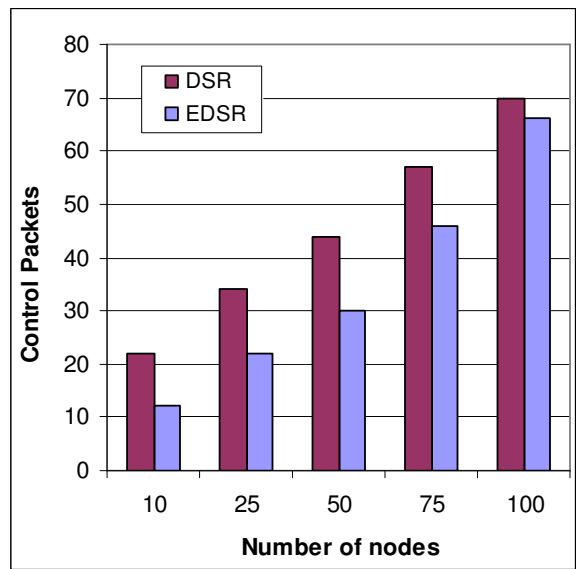

Figure 7. Comparison of DSR and E-DSR in case of reducing Control packets for a network of $10,25,50,75$ and 100 nodes. 


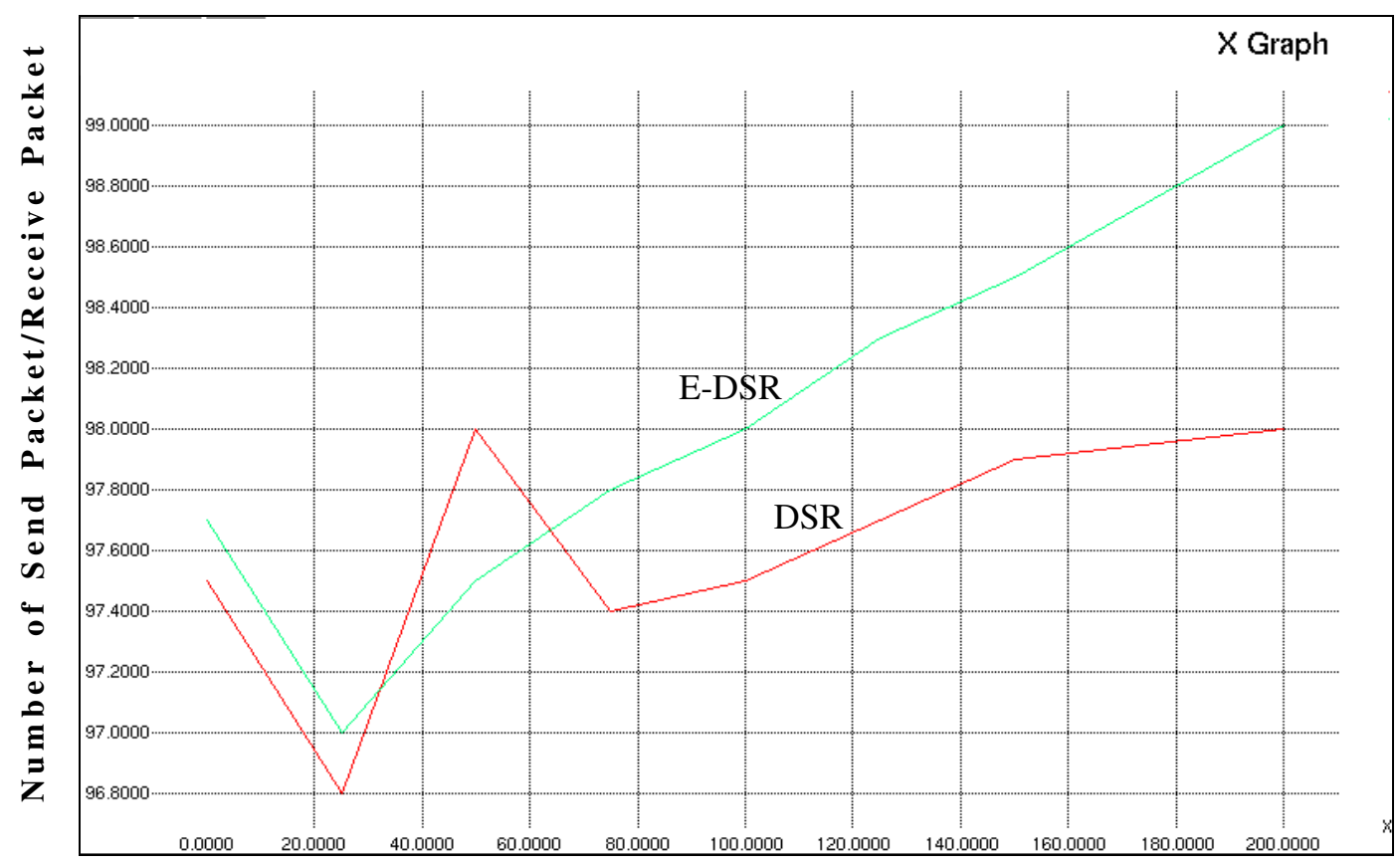

Time

Figure 8. Data delivery ratio of DSR and E-DSR for a network of 100 nodes

\section{REFERENCES}

[1] C.E.Perkins, (2001) "Ad hoc networking”, Addison-Wesley.

[2] J.M. Macker and S. Corson, (1997) "Mobile Ad Hoc Networks", IETF Working Group charter.

[3] O.H. Hussien, T.N. Saadawi, M.J. Lee, (12 Dec, 2005) "Probability Routing Algorithm for Mobile Ad Hoc Networks' Resources Management”, IEEE Journal on Selected Areas in Communications, vol. 23, no.

[4] D.B. Johnson and D.A. Maltz, (1996) "Dynamic Source Routing in Ad Hoc Wireless Networks", In Mobile Computing, edited by T. Imielinski and H. Korth, Chapter 5, Kluwer Academic Publishers, pp. 153-181.

[5] David B.Johnson, David A.Maltz,Yih Chun Hu, ( July, 2004) “The Dynamic Source Routing Protocol for Mobile Ad Hoc Networks(DSR)”, Internet Draft, draft-ietf-Manet-dsr-10.txt.

[6] C Perkins, E Belding-Royer and S. Das, (July 2003) "Ad hoc On-Demand Distance Vector (AODV) Routing", Request for Comments RFC 3561.

[7] Jyoti Raju, J.J.Garcia Luna Aceves, (October 2001) "Scenario based comparison of source_tracing and Dynamic Source Routing protocols for Ad Hoc Networks", ACM SIGCOMM Computer Communication Review Vol. 31, No.5.

[8] Charles E. Perkins, Elizabeth M. Royer, Samir R. Das and Mahesh K. Marina, (February 2001) "Performance comparison of two on-demand routing protocols for ad hoc networks", IEEE Personal Communication.

[9] Y.-C. Hu and D. Johnson, (Aug. 2000) "Caching Strategies in on demand Routing Protocols for Wireles Ad Hoc Networks", Proceedings of International Conference on mobile Computing and Networks. 
[10] M. Marina and S. Das, (April 2001) "Performance of Route Caching Strategies in Dynamic Source Routing" 21st International Conference on Distributed Computing Systems Workshops (ICDCSW '01), pp.0425.

[11] N. Ashokraj, C. Arun, K. Murugan (2008) "Route Cache Optimization Mechanism Using Smart Packets for On-demand Routing Protocol in MANET", International Conference on Information Technology, pp.141-146.

[12] USC/Information Sciences Institute, Network Simulator, at http://www.isi.edu /nsnam/ns/.

\section{Authors}

Sharmin Sultana received her MS and B.Sc. degrees in Computer Science and Engineering from University of Dhaka, Bangladesh, in 2009 and 2007 respectively. Now she is a Lecturer in Computer Science and Engineering at the Ahasanullah University of Science and Technology, Bangladesh. Her research interests include Networking and Communications, Mobile Networking, Digital Signal Processing, Artificial Intelligence and Genetic Algorithm.

Salma Begum received her MS and B.Sc. degrees in Computer Science and Engineering from University of Dhaka, Bangladesh, in 2009 and 2007 respectively. Now she is a Lecturer in Computer Science and Engineering at the Ahasanullah University of Science and Technology, Bangladesh. Her research interests include Ad-hoc Network, Wireless and Sensor Networking, Digital Image Processing.

Nazma Tara received her MS and B.Sc. degrees in Computer Science and Engineering from University of Dhaka, Bangladesh, in 2009 and 2007 respectively. Now she is a Lecturer in Computer Science and Engineering at The Peoples' University of Bangladesh, Bangladesh. Her research interests include Wireless Networking, Logic Synthesis and Design, Reversible Logic, Fuzzy Logic, Multiple-valued Logic.

Ahsan Raja Chowdhury received his MS and B.Sc. degrees in Computer Science and Engineering from University of Dhaka, Bangladesh, in 2005 and 2003 respectively. Now he is a Lecturer in Computer Science and Engineering at the University of Dhaka, Bangladesh. His research interests include Ad-hoc Network, Logic Synthesis and Design, Reversible Logic, Fuzzy Logic, Multiplevalued Logic, Genetic Algorithm, Bioinformatics and Evolutionally Algorithms.
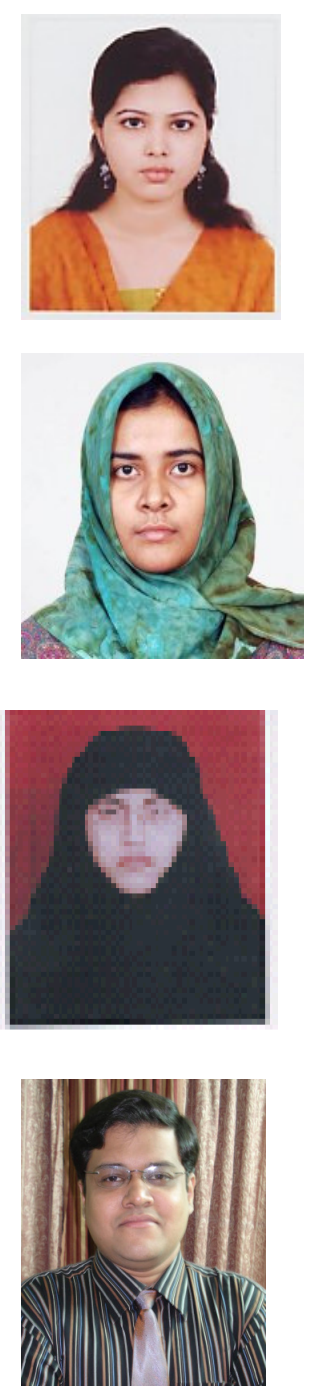